\title{
Design criteria of the bolometer diagnostic for steady-state operation of the W7-X stellarator ${ }^{\text {a) }}$
}

\author{
D. Zhang ${ }^{1, b)}$ R. Burhenn, ${ }_{1}^{1}$ R. Koenig, ${ }^{1}$ L. Giannone, ${ }^{1}$ P. A. Grodzki, ${ }^{1}$ B. Klein, ${ }_{1}^{1}$ \\ K. Grosser, ${ }^{1}$ J. Baldzuhn, ${ }^{1}$ K. Ewert, ${ }^{7}$ V. Erckmann, ${ }^{1}$ M. Hirsch, ${ }^{1}$ H. P. Laqua, ${ }^{1}$ \\ and J. W. Oosterbeek ${ }^{2}$ \\ ${ }^{1}$ Max-Planck Institut für Plasmaphysik, EURATOM Association, D-17491 Greifswald, Germany \\ ${ }^{2}$ Technische Universiteit Eindhoven, Den Doelch 2, 5612 AZ Eindhoven, The Netherlands
}

(Presented 19 May 2010; received 17 May 2010; accepted 10 July 2010;

published online 28 October 2010)

\begin{abstract}
A bolometric diagnostic system with features necessary for steady-state operation in the superconducting stellarator W7-X was designed. During a pulse length of $1800 \mathrm{~s}$ with an ECRH (electron cyclotron resonance heating) power of $10 \mathrm{MW}$, the components suffer not only from a large thermal load but also from stray radiation of the nonabsorbed isotropic microwaves. This paper gives an overview of the technical problems encountered during the design work and the solutions to individual problems to meet the special requirements in W7-X, e.g., component thermal protection, detector offset thermal drift suppression, as well as a microwave shielding technique.

(C) 2010 American Institute of Physics. [doi:10.1063/1.3483194]
\end{abstract}

\section{INTRODUCTION}

The stellarator W7-X has superconducting coils and is capable of steady-state operation. Long pulse discharges of $1800 \mathrm{~s}$ will be maintained by $140 \mathrm{GHz}$ microwave ECRheating with $10 \mathrm{MW}$ power in total. Plasma radiation is expected to lead to several $10 \mathrm{~kW} / \mathrm{m}^{2}$ average thermal loads on plasma facing components. In addition, the microwaves which are not sufficiently absorbed by plasma will be finally absorbed by plasma-facing components after a multiple reflection process. The latter is particularly harmful for components with higher rf-absorption coefficients and positioned close to the launching antenna. Diagnostics facing the plasma suffer from the high thermal power flux as well as from the microwave disturbance and need therefore careful considerations in design. ${ }^{1,2}$ The bolometer is especially sensitive to the thermal load from photons and to interference from microwaves. To avoid the highest microwave stray radiation flux near the antenna, all the planned bolometer cameras ${ }^{4}$ are allocated to ports at a distance from the antenna. This reduces the microwave radiation level to around $20 \mathrm{~kW} / \mathrm{m}^{2}$ from an expected level of $200 \mathrm{~kW} / \mathrm{m}^{2}$ in certain heating scenarios. ${ }^{3}$ Even so, the remnant stray radiation can still significantly influence the functionality of the bolometers. Using two cameras from the main bolometer system ${ }^{4}$ as examples, the paper gives an overview of the technical problems encountered during the design work and the solutions to individual problems to meet the special requirements in W7-X. In Sec. II the properties of the selected detectors will be described, laying a basis for initializing the design

\footnotetext{
Contributed paper, published as part of the Proceedings of the 18th Topical Conference on High-Temperature Plasma Diagnostics, Wildwood, New Jersey, May 2010.

b)Electronic mail: daz@ipp.mpg.de.
}

work. Topics related to the subcomponents are described in Sec. III Additional points and remarks will be discussed in Sec. IV.

\section{BOLOMETER DETECTOR}

Metal film resistive detectors ${ }^{5}$ and AXUV (absolute extreme ultraviolet) photodiodes are preferably utilized in the bolometric diagnostic for measuring plasma radiation in fusion devices. Both of them possess high spectral response for soft-x-rays. They have, however, their own shortcomings. The metal film has a high reflectance to visible light $(>400 \mathrm{~nm})$, while the photodiodes have a low, even unstable response to the UV range $(10-400 \mathrm{~nm})$, i.e., the socalled aging effect. ${ }^{6}$ We select the metal resistive detector as the main bolometer detector for W7-X in view of the advantages of its long-term, stable response, and especially, its high absorption coefficients in both UV $(>85 \%)$ and soft-xray range $(>95 \%)$, which cover almost the whole spectrum of the plasma radiation. Moreover, the metal film resistive detector allows an in situ Ohmic calibration. ${ }^{7}$

The gold film resistive detector with a $4 \mu \mathrm{m}$ thick gold absorber and a $7.5 \mu \mathrm{m}$ thick Kapton foil as substrate is our first choice. Its high sensitivity $(200 \mathrm{nW})$, reliability, and endurability have been confirmed at W7-AS and at other controlled fusion devices such as LHD and ASDEX Upgrade. For application at W7-X, care should be taken for avoiding detector damage due to overheating during both long pulse discharges as well as machine conditioning $\left(150{ }^{\circ} \mathrm{C}\right)$, since the maximum allowable working temperature of the Kapton-based film is around $130^{\circ} \mathrm{C}$. Suppression of the offset thermal drift $(100 \mu \mathrm{V} / \mathrm{K})$ is achieved by a water cooling system (see Sec. III B) to minimize the temperature rises of the bolometer camera housing and the detector holder. Additionally, a shutter will be used to perform measurements of the offset during a discharge (see 

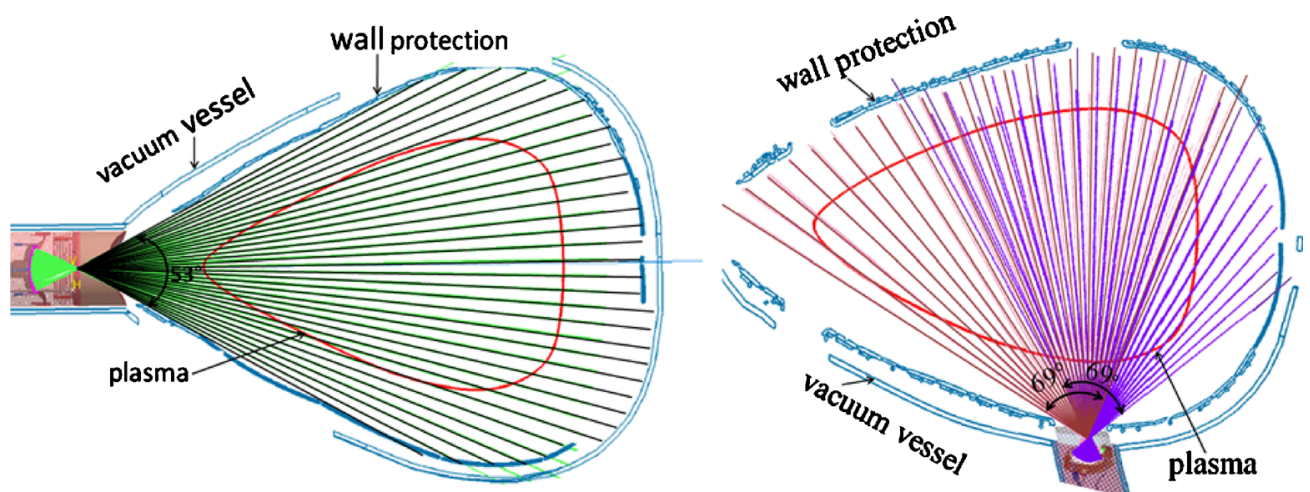

FIG. 1. (Color online) Lines of sight of HBC (left), consisting of 32-channel detectors, and VBC (right) possessing two subdetector arrays (20 channels each) viewing plasma through own apertures.

Sec. III D). An alternative candidate for the W7-X bolometer is the SiN-based Pt-resistive bolometer developed for the ITER. ${ }^{8}$ In comparison to gold, platinum and SiN have smaller interaction cross sections with neutrons. Therefore, they can better withstand the neutron irradiation levels expected in ITER. It follows the same principle as the gold-film detector, utilizing almost the same film holder. It is attractive particularly in its shorter response time and higher withstanding temperature $\left(>150{ }^{\circ} \mathrm{C}\right)$. A response time of $3 \mu \mathrm{s}$ is estimated for a $3 \mu \mathrm{m}$ thick SiN based Pt-detector, in comparison with that of $0.25 \mathrm{~ms}$ for a $7.5 \mu \mathrm{m}$ thick Kapton based Au-detector, according to the relationship between the thermal diffusion time in a substrate with the thickness $\mathrm{L}$ and the thermal diffusivity $D$, i.e., $\mathrm{L}^{2} /(2 \mathrm{D})$. Such a short response time is of course desirable for resolving fast phenomena in SOL (scrape-off layer)-transport time scales such as ELMs (edge-localized-mode), detachment, and MARFEs (multi faceted radiation from the edge). Further developments to improve its endurability are necessary and are already underway. ${ }^{9}$

\section{DESIGN}

\section{A. Line of sight}

The most important design criterion of the lines of sight of the bolometer is to obtain a high spatial resolution and a good coverage over the plasma cross section to be investigated. The lines of sight are collimated through a rectangular aperture, whose dimensions together with the distance between the aperture and the detectors determine the camera viewing angle and the widths of lines of sight. Since the applicable space in the port to install the detector array is limited, a fan-shaped line of sight set is designed, with all detectors (four-channel bolometer heads) being arranged equidistantly to the aperture center for a maximum power flux reception. A 32-channel detector array with an aperture of $5 \times 10 \mathrm{~mm}^{2}$ is designed for the horizontal bolometer camera (HBC). A spatial resolution of $\sim 5 \mathrm{~cm}$ (the width of the line of sight at the magnetic axis) and a desired viewing angle of $53^{\circ}$ are achieved, which actually covers the whole torus cross section, hence, guaranteeing the coverage over the varied plasma cross section with different magnetic configurations (see Fig. 1, left). A secondary detector array with different detectors, being adjustable to be positioned sym- metrically around the slit axis with respect to the above mentioned main one, is planned to be integrated to the camera gradually with different detectors. ${ }^{4}$ As a first step, four blind channels will be installed in it for eliminating offsets and drifts. Later, detectors using Be-foils as optic filters can be mounted to allow detection of high energetic photons and provide additional information on heavy impurity concentration in the plasma center.

For the vertical bolometer camera (VBC) a viewing angle of around $120^{\circ}$ is required. A good coverage of the plasma cross section is achieved by structuring the camera as two subdetector arrays (20 channels each) viewing plasma through their own apertures $\left(5 \times 10 \mathrm{~mm}^{2}\right.$ each). They are displaced toroidally (see Fig. 2) as close as possible for keeping the observed plasma unchanged. An optic separator positioned between the two arrays is designed to distinguish the radiation sources (see Fig. 2, left).

As the flux surfaces in a stellarator are not axisymmetric, the toroidal extension of the lines of sight needs to be restricted so that the displacement of the magnetic axis lies in the range of the spatial resolution. The toroidal dimension of the slit aperture is determined by the required spatial resolution and signal/noise ratio.

\section{B. Thermal protection and thermal drift suppression}

The bolometer cameras must be positioned properly to get the required viewing angles. The VBC must be placed

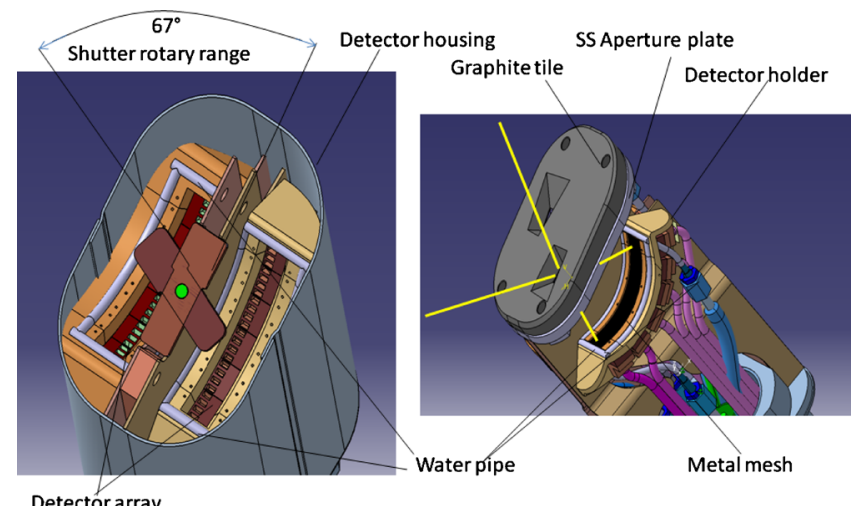

FIG. 2. (Color online) Overview of the vertical camera head design. (Left) The two subdetector arrays mounted on the two water cooled detector holders, separated with optic baffle. (Right) Graphite tile capped in front of the stainless steel aperture plate as thermal protection. 
close to the opening on the wall protection structure (see Fig. 1 , right). The thermal load onto the aperture can reach several $100 \mathrm{~kW} / \mathrm{m}^{2}$. A water cooling system is implemented into the aperture plate and a structured graphite tile is added as additional thermal protection (see Fig. 2, right). An intermediate graphite foil is pressed between the graphite tile and the stainless steel (SS) aperture plate for enhancing the thermal contact.

A water cooling structure is also embedded into the detector holder, as shown in Fig. 2, to minimize temperature drift during long-pulse discharges. The detector holder is made of $\mathrm{CuCrZr}$, as its higher thermal conductivity ( 25 times higher than that of SS) reduces temperature gradients due to the difference of local power depositions. Two Pt100 resistive thermometers will be installed at different positions in the holder to monitor the temperature gradient and change. Thermal analysis of the structures with ANSYS has been performed assuming the $\mathrm{HBC}$ and $\mathrm{VBC}$ are exposed to power flux densities of 50 and $400 \mathrm{~kW} / \mathrm{m}^{2}$, respectively. For an exposure time of $1800 \mathrm{~s}$, the SS aperture plates of the both cameras maintain maximum local temperatures below $150{ }^{\circ} \mathrm{C}$ with cooling, instead of $900{ }^{\circ} \mathrm{C}$ without cooling, while all the detector holders keep their temperatures lower than $40{ }^{\circ} \mathrm{C}$ with cooling, instead of over $150{ }^{\circ} \mathrm{C}$ without cooling.

\section{Microwave shielding system}

\section{Microwave impact}

Microwave reaching the metal film absorber will induce eddy currents in several skin depths and heat the absorber. Gold film has a skin depth of $0.2 \mu \mathrm{m}$ for $140 \mathrm{GHz}$ microwave. The absorption factor of an isotropic microwave is estimated to be $0.1 \%$, which is confirmed in laboratory utilizing the gold film detector exposed to a collimated microwave beam launched by a horn-shaped antenna. Single-path microwave absorption is then demonstrated. In the case of the camera exposed to isotropic microwave stray radiation in W7-X, power flux going through the aperture is first reduced to large extent. However, multiple reflection in the cavitylike detector housing enhances the effective power flux density (PFD), which can be expressed as $\mathrm{P}_{0} \mathrm{~S}_{\mathrm{a}} / \mathrm{SUM}\left(\mathrm{S}_{\mathrm{c}, \mathrm{i}} \alpha_{\mathrm{i}}\right)$, where $\mathrm{P}_{0}$ is the input power flux density at the aperture, $\mathrm{S}_{\mathrm{a}}$ the aperture area, $S_{c, i}$ the surface of the elements forming the cavity, and $\alpha_{\mathrm{i}}$ the corresponding microwave absorption coefficient. According to the designed camera structure and material composition, PFD in the camera maintains the same order of amplitude as $\mathrm{P}_{0}$. For PFD of $20 \mathrm{~kW} / \mathrm{m}^{2}$ the resultant detector absorbed power is $0.1 \mathrm{~mW}$, comparable to the plasma radiation induced one $(0.1-1.0 \mathrm{~mW})$. Proper shielding is therefore necessary and details of achieving the required level of attenuation are discussed in the next section.

\section{Microwave shielding and trapping}

Microwave shielding can be achieved by mounting a conductive wire-mesh in front of the detector. The opening of the mesh $w$ should be smaller than half of the wavelength $\lambda$. Furthermore, the mesh wires should have a finite thickness (diameter) $\phi$ of sufficiently low resistance for an effective
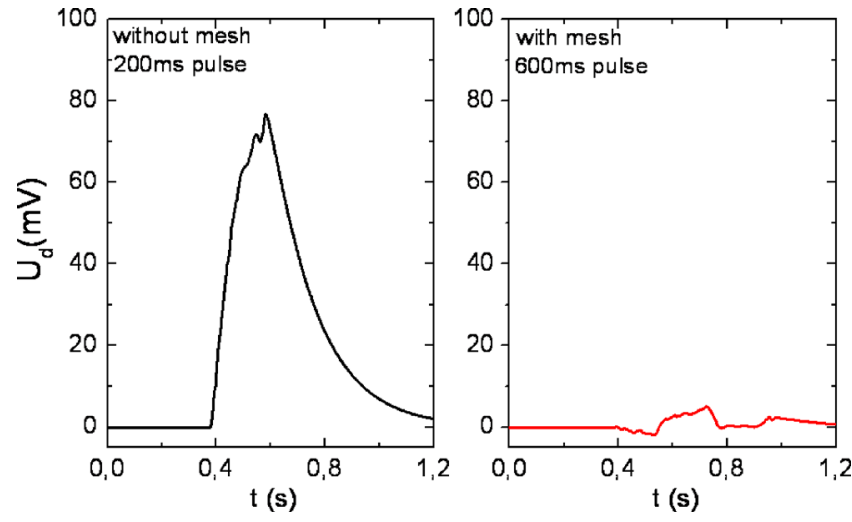

FIG. 3. (Color online) Comparison of bolometer signals tested in MISTRAL in the cases without (left) and with mesh screening (right).

microwave reflection on one hand and for avoiding melting on the other. This reduces, however, the transmission factor of the photons onto the bolometer. The signal strength of the bolometer is proportional to the percentage of the open area of the mesh, i.e., $w^{2} /(w+\phi)^{2}=1(1+\phi / w)^{2}$. The larger the ratio $\phi / w$, the smaller the photon transmission factor and the higher the microwave shielding efficiency. Thus, a compromise between the microwave shielding and the optic transmission must be made. In the laboratory, meshes with different combinations of $w(<0.5 \mathrm{~mm})$ and $\phi$ have been tested with $140 \mathrm{GHz}(\lambda / 2=1.1 \mathrm{~mm})$ microwaves. The measurements indeed show a dependence of the transmission factor on both $w$ and $\phi$. A metal-mesh with $\phi=90 \mu \mathrm{m}$ and $w$ $=0.24 \mathrm{~mm}$ is selected for further tests in view of its low microwave transmission factor of $5 \%$ and high optic transmission factor of 53\%. Further optimization of the mesh will aim to improve its thermal and electrical conductivity. A copper-mesh or bronze-mesh might be the best candidate. Integration of the mesh into cooled detector holder is already designed (see Fig. 2, right).

Experiments for testing the microwave-induced effects on the bolometers have been carried out under even more serious conditions than in W7-X. A prototype of the W7-X bolometers has been tested in the MISTRAL (Refs. 10 and 11) chamber which provides an isotropic, nearly homogeneous microwave background. For a total input power to the MISTRAL of $200 \mathrm{~kW}$, the bolometer absorbs a power amount of $\sim 10 \mathrm{~mW}$, which is almost 100 times larger than that expected in W7-X. After mounting the selected mesh in front of the detector, the power flux onto the detector is reduced by a factor close to 20. A comparison of the detector signal with and without mesh screening is shown in Fig. 3. This confirms the mesh shielding effectiveness obtained in laboratory conditions. Thus, the microwave induced signal at $\mathrm{W} 7-\mathrm{X}$ is expected to be reduced to around $10 \%$ of the plasma induced one. For further reducing the microwave impact, a ceramic absorber $\left(\mathrm{TiO} / \mathrm{Al}_{2} \mathrm{O}_{3}\right)$ coating on the inner surface of the detector housing is planned in order to reduce multipath reflectance of the microwaves in the camera housing. An alternative method is to use a set of properly spaced metal plates, being of lamella-like structure with ceramic absorber coating to prevent the microwaves in the camera housing reaching the bolometer absorber. 


\section{Multifunction of shutter}

A UHV (ultra high vacuum) compatible pneumatic driven shutter has been designed. Remote control by an (air control) electrical valve is necessary because of the restricted accessibility to the diagnostics during experiments. Closure of the shutter allows to perform in situ calibration of detectors and offset measurement between experiment segments as well as to protect the detectors against contamination during conditioning.

\section{ADDITIONAL ISSUES}

W7-X ports connect the vacuum vessel and the outer cryostat vessel, have different lengths $(1.5-2.8 \mathrm{~m})$, and provide limited spaces $(150-300 \mathrm{~mm})$ for housing bolometers. Bellows in the port wall compensate the relative thermal movement of the two vessels. The bolometer cameras will be fixed on the rigid part of the port wall connected to the vacuum vessel for mechanical stability. The bolometers are designed as plug-in elements, allowing installation and removal from outside of the machine. For plug-ins of around 2 $\mathrm{m}$ length, any inaccuracy in the port axis and the camera positioning are potential error sources leading to systematic deviations of lines of sight from their original design. Appropriate compensation strategies have been already taken into account.

${ }^{1}$ R. König, J. Baldzuhn, W. Biel, C. Biedermann, R. Burhenn, S. Bozhenkov, J. Cantarini, H. Dreier, M. Endler, H.-J. Hartfuss, D. Hildebrandt, M. Hirsch, M. Jakubowski, R. Jimenez-Gomez, G. Kocsis, P. Kornejev, M.
Krychowiak, H. Laqua, M. Laux, J. W. Oosterbeek, E. Pasch, T. Richert, W. Schneider, B. Schweer, J. Svensson, H. Thomsen, A. Weller, A. Werner, R. Wolf, D. Zhang, and D. Zoletnik, Rev. Sci. Instrum. 81, 10E133 (2010).

${ }^{2}$ R. Burhenn, J. Baldzuhn, W. Biel, H. Dreier, M. Endler, R. J. Gomez, K. Grosser, H. J. Hartfuss, D. Hildebrandt, M. Hirsch, R. König, P. Kornejev, M. Krychowiak, H. P. Laqua, M. Laux, J. W. Oosterbeek, E. Pasch, W. Schneider, H. Thomsen, A. Weller, A. Werner, R. Wolf, and D. Zhang, "Diagnostics development for steady state operation of the stellarator Wendelstein 7-X," International Conference on Plasma Diagnostics, Pontà-Mousson, France, 12-16 April 2010 [Contrib. Plasma Phys. (to be published)].

${ }^{3}$ H. P. Laqua, V. Erckmann, and M. Hirsch, Proceedings of the 28th EPS Conference on Controlled Fusion and Plasma Physics, Madeira, 2001, Vol. 25A, pp. 1277-1280.

${ }^{4}$ D. Zhang, L. Giannone, B. Klein, and W7-X Team, Proceedings of the 34th EPS Conference on Controlled Fusion and Plasma Physics, Warsaw, 2-6 July 2007.

${ }^{5}$ K. F. Mast, J. C. Vallet, C. Andelfinger, P. Betzler, H. Kraus, and G. Schramm, Rev. Sci. Instrum. 62, 744 (1991).

${ }^{6}$ R. Korde, J. Cable, and L. Canfield, IEEE Trans. Nucl. Sci. 40, 1655 (1993).

${ }^{7}$ L. Giannone, K. Mast, and M. Schubert, Rev. Sci. Instrum. 73, 3205 (2002).

${ }^{8}$ L. Giannone, D. Queen, F. Hellman, and J. C. Fuchs, Plasma Phys. Controlled Fusion 47, 2123 (2005).

${ }^{9}$ H. Meister, T. Eich, L. Giannone, M. Kannamüller, A. Kling, J. Koll, T. Trautmann, ASDEX Upgrade Team, P. Detemple, and S. Schmitt, Rev. Sci. Instrum. 81, 10E132 (2010).

${ }^{10}$ M. Hirsch, J. W. Oosterbeek, R. Jimenez, J. Baldzuhn, M. Endler, M. Laux, D. Zhang, H. P. Laqua, F. Noke, F. Purps, and K. Ewert, "Qualifying plasma diagnostics for a high-power microwave background radiation of ECR-heated discharges." International Conference on Plasma Diagnostics, Pont-à-Mousson, France, 12-16 April 2010 [Contrib. Plasma Phys. (to be published)].

${ }^{11}$ S. Ullrich, H. J. Hartfuss, M. Hirsch, and H. Laqua, Stellarator News 98, 2005, http://www.ornl.gov/sci/fed/stelnews. 